\title{
Sensor Network Deployment For Agronomical Data Gathering in Semi-Arid Regions
}

\author{
Prabhakar T V , N V Chalapathi Rao, Sujay M S, Jacques Panchard, H S Jamadagni, Andre Pittet. \\ Centre for Electronics Design and Technology \\ Indian Institute of Science, \\ Bangalore, India \\ [tvprabs, nvcrao, mssujay]@cedt.iisc.ernet.in, Jacques.panchard@epfl.ch, [hsjam, apittet]@cedt.iisc.ernet.in
}

\begin{abstract}
We share our experience in planning, designing and deploying a wireless sensor network of one square kilometre area. Environmental data such as soil moisture, temperature, barometric pressure, and relative humidity are collected in this area situated in the semi-arid region of Karnataka, India. It is a hope that information derived from this data will benefit the marginal farmer towards improving his farming practices. Soon after establishing the need for such a project, we begin by showing the big picture of such a data gathering network, the software architecture we have used, the range measurements needed for determining the sensor density, and the packaging issues that seem to play a crucial role in field deployments. Our field deployment experiences include designing with intermittent grid power, enhancing software tools to aid quicker and effective deployment, and flash memory corruption. The first results on data gathering look encouraging.
\end{abstract}

Keywords-COTS hardware, MICA2 motes, Antenna masts, TinyOS, Sensor deployment.

\section{INTRODUCTION}

Studies in [1] reveal that by 2025 , about 2.7 billion people, nearly one-third of the expected world population, will live in regions facing severe water scarcity. Asia and sub-Saharan Africa containing the most populated and poorest regions will be severely affected.

The opinion about usage of water is sharply divided. While environmentalists recommend that water usage should be down by $10 \%$ to save the existing ecosystem, the agriculturists argue that $15-20$ percent increase in the coming 25 years is required to maintain food security, reduce hunger and rural poverty.

Among the rural agriculture dependant population, marginal farmers are those whose land holdings are about 1 hectare and represent a group both significant in number of individuals and in relation to the environmental impact this group have through its agricultural practices. For example, in Pavagada (Karnataka, India) taluk, the centre of our project, $54 \%$ are marginal farmers with about $36 \%$ of the land holding. Unlike the rich farmers, they can neither afford to pay for powered irrigation, nor can protect the crop and cattle in the event of pests and diseases. Thus, these farmers depend heavily on the seasonal rainfall. The semi-arid region in our project site is one where the rain fall is about $500 \mathrm{~mm} /$ year with uneven distribution over 6 months. The region is also associated with high annual variation of about $250-1000 \mathrm{~mm}$, with poor water retention coupled with high run-off.

We believe that COMMONSenseNet (Community Oriented Management and Monitoring Of Natural Resources through Sensor Networks) project is an innovative use of wireless sensor network for the benefit of the marginal farmer in the semi-arid region. We hope that the network's constant monitoring of crop condition and providing reliable information will help the farmers use this technology for taking appropriate decision on standing crops. One of the features is that the community will use locally available talent for sustaining the collection of data, timely analysis and dissemination. The deployment will use local infrastructure, materials, fabrication and import only the bare minimum high technology hardware.

\section{USER SURVEY AND REQUIREMENTS}

User surveys conducted as part of this project have indicated that farmers are convinced that a better knowledge of the physical parameters related to the field would help them refine their strategies and practices.

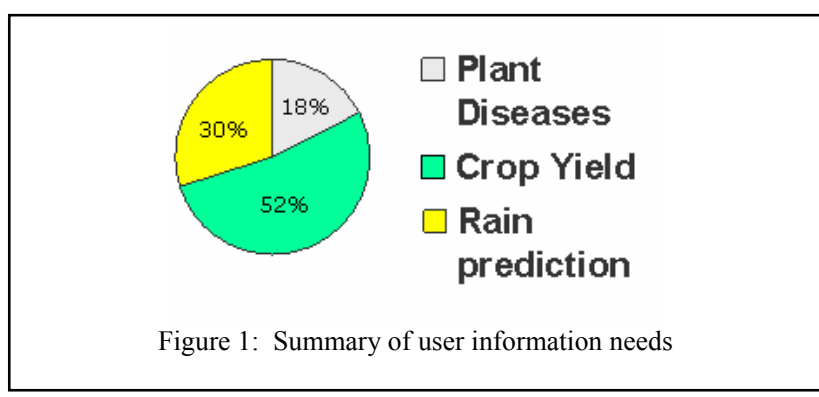

Figure 1 gives a summary of the farmer's information needs. Over $50 \%$ of the farmers in a total of 300 families across 5 villages need information about crop yield. The remaining farmers need information about rainfall and pest attacks that lead to plant diseases.

With a view of meeting the user's information needs, a set of environmental data of immediate use to farmers such as soil moisture, temperature, pressure and humidity is gathered using wireless sensor network technology. The data is made available to agronomists, hydrologists and agriculturalists for data 
processing. The final information can be used by marginal farmers.

The semi-arid region for the project is chosen to be Chennakesavapura and four other surrounding villages in the Pavagada taluk. The requirement is to cover 25 square kilometre area with the soil moisture sensor density of about 360 sensors/hectare and 10 sensors/hectare for monitoring temperature, pressure and humidity. The coverage area is divided into multiple clusters with a Field Station Data Aggregation Unit (FDA) located almost in the centre of the proposed deployment area. Each sensor in a cluster gathers data and provides it over a multihop to a Field Data Collection Unit (FDC).

Concerning the infrastructure, it is initially proposed to use Commercially Off the Shelf (COTS) hardware to build the monitoring network. Free software tools with Linux operating system are envisaged. Since the sensor nodes are currently not suitable for long range communication, long haul connectivity between FDA's and FDC's is achieved by using Wi-Fi's Wireless Distribution System (WDS) [2] technology in bridge/repeater mode. Crossbow packaged MICA2 [3] motes are identified for interfacing sensors and associated communication.

\section{PREPARING FOR A DEPLOYMENT}

\section{a) Data Gathering:}

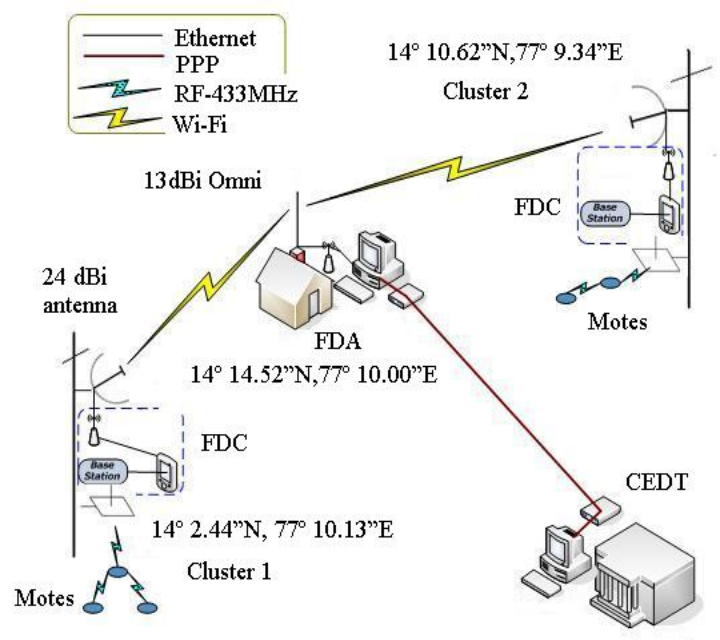

Figure 2: Big picture of Data gathering with FDA and FDCs

Figure 2 shows the prototype deployment at the project site. It comprises of two clusters and associated COTS hardware for both the FDA and FDC units. Each cluster has a 24dBi directional antenna pointing towards the FDA unit's $13 \mathrm{dBi}$ omni directional antenna.

Data from FDCs is transferred to FDA over a Wi-Fi link. In the event of link failure, since data is also stored on local storage within each FDC, it is transferred over a FTP connection upon request from a FDA.To transfer data from FDA to our laboratory in CEDT, we are currently using a dialup V.92 Modem.

\section{b) Software architecture:}

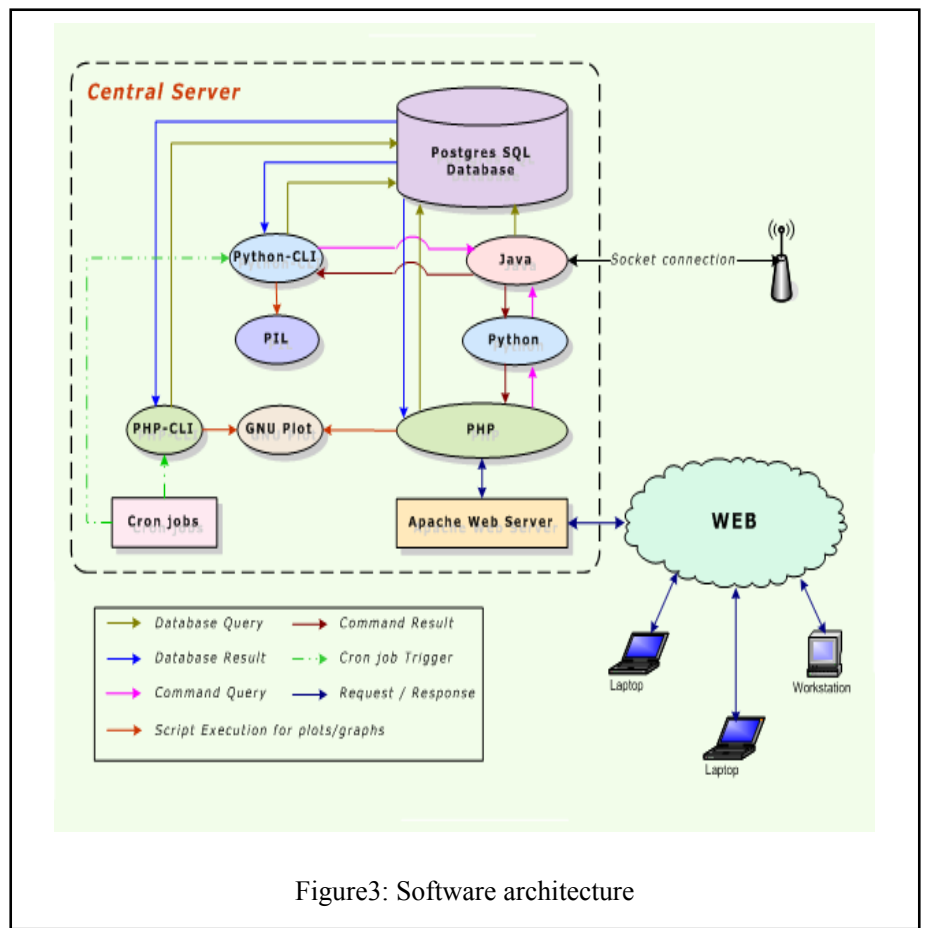

Figure 3 shows the software architecture for the data gathering network. It comprises of software applications such as Apache web server and PostGres SQL database server that use tools such as Java, Python and PHP to drive them towards data gathering, storage and retrieval.

The java tool runs a serial forwarder TCP application that receives data over a server port. The data packet together with a time stamp is stored in the database. Python is used for providing the data view graphs over the web interface as well as for displaying the routing tables.

\section{c) Range measurements:}

Range measurements [4] were carried out on Mica2 motes at the project site. Since the proposed deployment has to work in a multihop paradigm, it becomes imperative to determine the usable radio communication distance between two sensors. The experiments were conducted in an open plain land with antennae placed at a height of 1.2 meters from the ground level. Transmit power levels were set to $-10,0$ and $10 \mathrm{dBm}$. The data rates at each power level were 1,5 and $10 \mathrm{kbps}$. A packet transmission consisted of 8 bytes of preamble, 2 bytes of SYNC and 34 bytes of data. The total number of packets (n) to be transmitted was computed using the equation:

$$
n=\frac{1000}{p^{* l}}
$$

The goal is to sample at least 1000 packets to get the RSSI readings and $\mathrm{p}$ is the probability of bit error applied prior to making the measurements. We fix the p value to 0.001 and $l$ is 
the total number of bits in a packet $(34 * 8)$. Using equation 1.1 a total of 3676 packets were transmitted at each power level

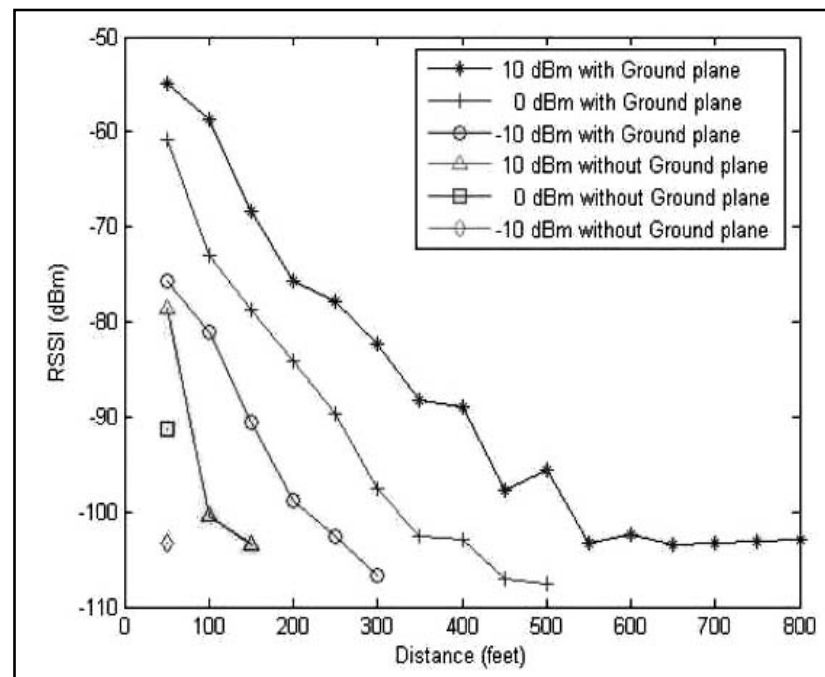

Figure 4: Range Measurement with Mica2

Figure 4 shows the results of the achieved communication range for a radio sensitivity of $-100 \mathrm{dBm}$. Using the ground plane $(\lambda / 4)$ and transmit power of $+10 \mathrm{dBm}$ one may deploy sensors up to about 550 feet.

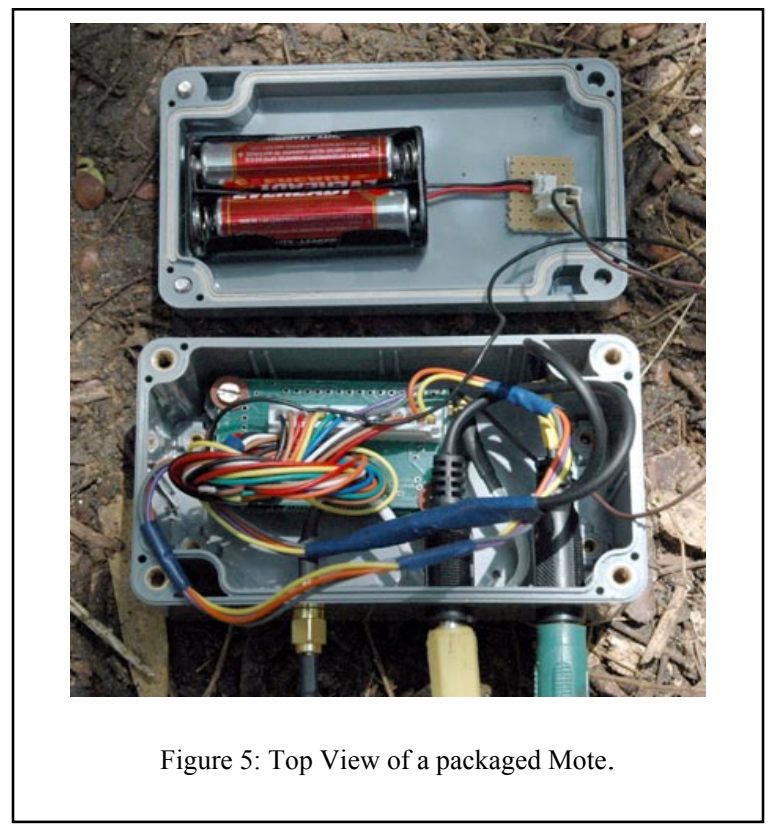

d) Packaging issues:

Essential packaging requirements include Index of protection (IP) specifications of IP 65 which ensure that the sensors, hardware electronics, masts and all other systems are not affected by moisture, sunshine, mild impacts, vegetation, macro and micro biological agents, common chemical weedicides and pesticides.
A trial deployment was carried out in CEDT. This deployment may be viewed at http://www.commonsensenet.in For the ground plane, the original specifications called for a copper plate. This was replaced with a very inexpensive GI mesh of appropriate grid spacing based on the dipole length. Hermetically sealed IP65 enclosures were selected from stock items and the required terminations for the sensors and the antenna were carried out. The masts were fabricated locally (using common materials) as per design provided by the project team. Figure 5 shows the motes packaging with appropriate enclosure and termination.

Figure 6 shows a sealed enclosure with FDC COTS hardware which has the following components: 1) Crossbow's MIB510 board which switches the received packets to the UART. 2) PXA 255 based single board computer running embedded Linux. This board is used for receiving packets from the MIB510's UART and switching them to the Ethernet port for long haul transmission using Wi-fi in WDS mode. 3) Access point to provide $\mathrm{Wi}$-fi connectivity.

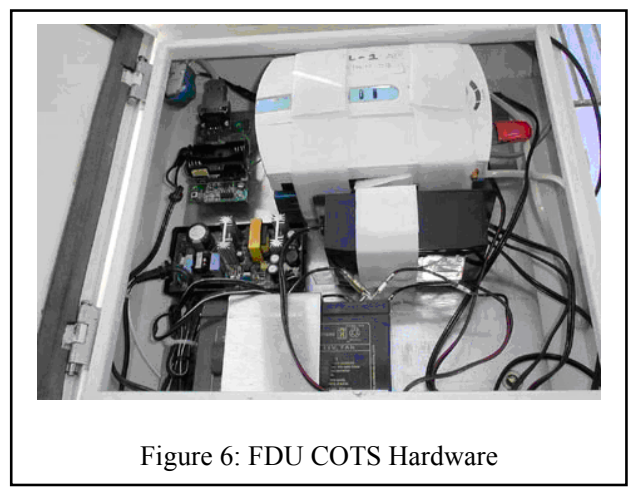

Power is drawn from $220 \mathrm{~V}$ AC mains and fed to the battery charger circuitry. A $12 \mathrm{~V}, 28 \mathrm{~A}-\mathrm{h}$ battery is charged to provide the necessary DC voltages using a DC-DC converter.

\section{DEPLOYMENT EXPERIENCES}

The Electronics hardware (Mica2 motes) along with soil moisture sensors were buried underground in IP65 enclosures procured locally. The others such as temperature, barometric pressure and relative humidity sensors were mounted above the ground on suitably designed mote antenna masts. The mote antenna has to be at a height to ensure near- line-of-sight.

The FDC requires about 25 watts of power. Grid Power supply is intermittent and sometimes stays off for a couple of days. However the units need to be powered up all the time. To facilitate this, regular 12V 28A-h SMF lead acid battery power pack was made. The whole system was split into the power supply module and the FDC module. Two separate IP55 outdoor pole mounted boxes were designed and fabricated. The design is such that very commonly used materials and fabrication processes are needed.

The normal power transmission in the Indian rural areas uses only a 3 wire $400 \mathrm{~V}$ AC system. This is made to ensure that consumers do not use the unmetered 3 Phase power for domestic use. The system charger needed a 1 P\& N 230V 
scheme; to facilitate this local false neutral was generated from the ground. Figure 7a shows the point where the phase alone is tapped from the distribution transformer. Figure $7 \mathrm{~b}$ shows the point where the neutral is generated locally and connected directly to the charger unit.
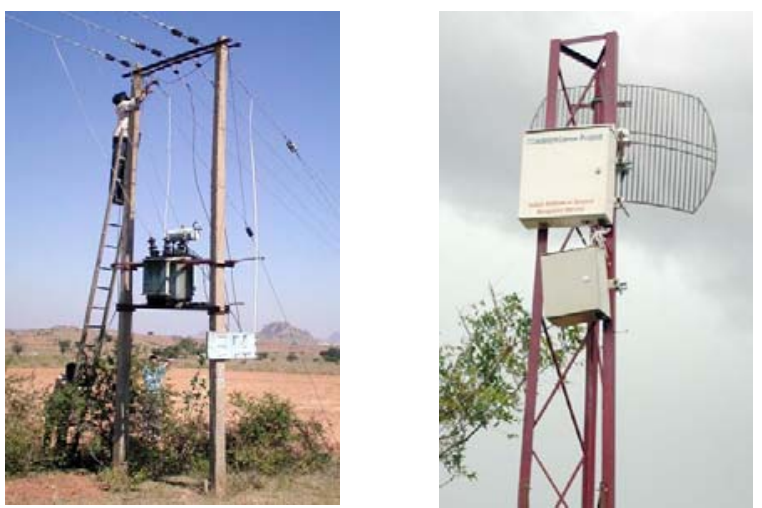

Figure 7a: AC Phase tap point. Fig7b: AC Neutral generated locally and connected to charger unit

The FDC (Figure 7b) was mounted on a 15 meter tower, locally fabricated. The FDA's Omni directional receive antenna is mounted at about 20 Meters above ground on one of the local farmers roof top and is expected to oversee a large area. Thus the FDA base station can pick up signals as and when more FDCs are added.

Our initial trials with wood masts failed, the wood being attacked by termites and the antenna losing its direction due to wind. Finally a heavy $100 \mathrm{Kg}$ steel structure was fabricated once again from locally available materials mainly mild steel construction stock.

During the sensor node deployment, we enhanced an existing network sniffer application called TOSBase application since we did not have high end equipment such as spectrum analyzer and field strength meter. The new application provides the user deployment friendly messages by interpreting the broadcast messages issued by active sensors. These messages include number of neighbors seen by a node including their radio proximities in terms of receive estimates and the number of hops to reach the FDC.

With an objective to save battery power of motes, the duty cycle was set to $28.57 \%$. To further save battery power of hop nodes, we modified the embedded code [5] to detect the presence of a sensor. By this method, generation of application packets by hop nodes was avoided.

Initially, the sensor was programmed to issue a data packet every 5 minutes with a route update control packet arriving every 3 minutes. Since this duration was found to be inconvenient for quick deployment, we had to modify the embedded code to transmit data packets once every 15 seconds for the first two hours and then switch back to transmit data packets every 5 minutes. This helped us to debug connectivity problems faster. We found that most often the RF SMA connectors between the RF output of the mote and the antenna was snapping due to weight of the soil. For this, we tried to pack them suitably. We finally found that a reliable connection is possible only if the mote was placed above the ground. Another reason to shift the motes above the ground was due attack by rodents nibbling away the buried RF cable.

Another problem we are currently facing is the flash memory corruption [6] of the embedded code .The type of flash on mica2 is a variant of the NOR flash called AT45DB. Flash corruption leads to either continuous rebooting ("D6" in all memory locations) of the node or a complete frozen state ("FF" in all memory locations). We did not encounter this problem in our prototype deployment probably due to the presence of thick vegetation and also our constant monitoring of mote battery voltages. We suspect that low battery voltage, UV light exposure and lightening seem to cause this problem. A sample of the corrupted flash memory is shown in figure 8 . The flash contents were found to contain "FF" and "D6" in about 250 contiguous memory locations.

\section{Section I}

S11340C0813041F08FB7F894282F33278DE0809307 S11340D01E022FBF81E090E008958F929F92AF92CD S11340E0BF92CF92DF92EF92FF920F931F93CF93E1 S11340F0DF93CDB7DEB7C054D0400FB6F894DEBF1F S1134100FFFFFFFFFFFFFFFFFFFFFFFFFFFFFFFFBB S1134110FFFFFFFFFFFFFFFFFFFFFFFFFFFFFFFFAB S1134120FFFFFFFFFFFFFFFFFFFFFFFFFFFFFFFF9B S1134130FFFFFFFFFFFFFFFFFFFFFFFFFFFFFFFF8B S1134140FFFFFFFFFFFFFFFFFFFFFFFFFFFFFFFF7B S1134150FFFFFFFFFFFFFFFFFFFFFFFFFFFFFFFF6B S1134160FFFFFFFFFFFFFFFFFFFFFFFFFFFFFFFF5B Section II

S11344C000000000000000009598939A000000008E S11344D000000000959A000000000000000095987C S11344E0C13069F493980000000000000000959A20 S11344F000000000000000009598939A1DC0C2308F S1134500D6D6D6D6D6D6D6D6D6D6D6D6D6D6D6D647 S1134510D6D6D6D6D6D6D6D6D6D6D6D6D6D6D6D637 S1134520D6D6D6D6D6D6D6D6D6D6D6D6D6D6D6D627 S1134530D6D6D6D6D6D6D6D6D6D6D6D6D6D6D6D617 S1134540D6D6D6D6D6D6D6D6D6D6D6D6D6D6D6D607 S1134550D6D6D6D6D6D6D6D6D6D6D6D6D6D6D6D6F7

Figure 8: Two sections of corrupted flash memory

Figure 8, shows the two sections of the corrupted flash memory. The last byte in the record indicates the checksum field. It is computed whenever flash is loaded or retrieved. It can be observed that the checksum field matches with the corrupted portion of flash memory.

During monsoon and other times when there is thunder and lightening in the skies, the telephone exchange in the Village is shutdown. Since this affects data retrieval from the FDA to our laboratory, we explored the possibility of using a CDMA operator's cellular network. We found that the modem inside the CDMA handset (LG 2030) was unable to pick data calls when dialed from an external V.92 modem. Furthermore, the CDMA handset can only respond to PPP negotiation from the CDMA operator. 


\section{FIRST RESULTS}

For our results on soil moisture, we have used the ECHO20 probes [7]. We compare the soil moisture values in our backyard with rain data obtained from a standard rain guage. Figure 9 shows the sensitivity of the soil moisture probe to the rainfall. Also, moisture retention in the soil can be observed.

During the dry days (month of May, July, September) the moisture tends to fall off indicating the absence of rainfall. The occurrence of rainfall which leads to an increase in the moisture content can be observed during the days of June and late August. Agricultural scientists may be able to suggest suitable cropping schedules after a profound study of this data where traditional rainfall data may not be much fruitful.

Figures 10 and 11 depict the pressure and temperature values sensed by our monitoring unit and a standard weather monitoring system. The sensirion sensors [10] mounted on the motes seem to be sensitive enough and closely follow a standard weather monitoring station.

\section{CONCLUSIONS AND FUTURE WORK}

Sensor network deployment for reliable data gathering has to be carefully planned and executed. Issues of local topography have a direct bearing on the deployment.

For our application scenario we found that the Mica2 motes are limited in communication range. After evaluating alternate platforms we think that Tiny node platform from shockfish [8] may be suitable. The tiny node has a low power 16 bit MSP430 micro controller from Texas Instruments and Xemics's XE1205 radio. The data sheet specifies a range of $1.8 \mathrm{~km}$ for a $15 \mathrm{dBm}$ transmission power. The radio supports advanced features such as hardware computation of received signal strength indicator (RSSI), pattern recognition block and buffered mode of operation which reduces the processor overhead. The MSP430 controller has a 12 bit Analog to Digital converter.

Future work is being carried out on replacing the existing FDC with custom hardware built in-house. We are exploring the use of standard System On Modules (SOM) with interface to daughter cards to provide the complete functionality. The advantages are low power operation and replacing COTS hardware for reducing costs while scaling to wide deployments.

Another development we are having in mind is to develop a camera interface for our sensing units. We hope to transmit still images and low frame rate video of the leaf during pest attacks.

We also need to look at alternate links to connect FDA to the internet. One possibility is to approach a broadband service provider to avail wireless broadband such as WiMax Services.
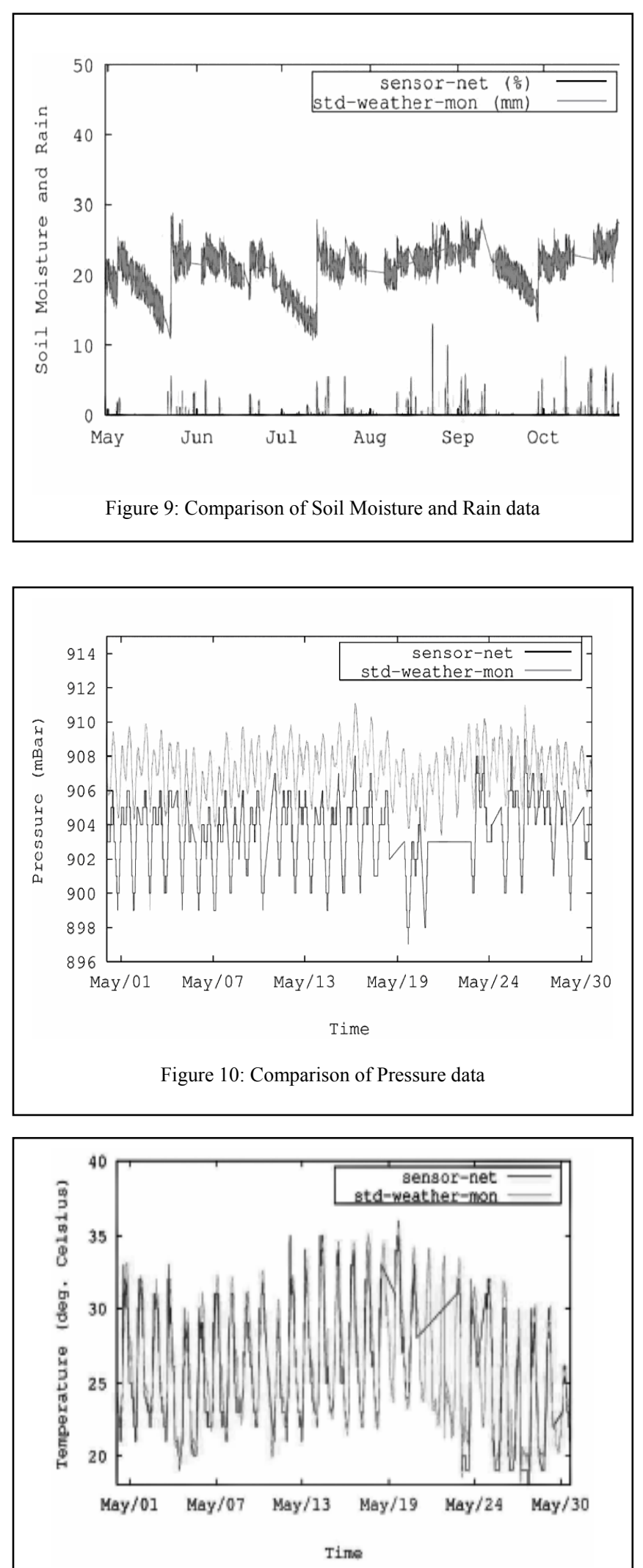

Figure 11: Comparison of Temperature data. 


\section{ACKNOWLEDGMENTS}

We thank Mr. Seshagiri Rao of Chennakesavapura trust for providing all the support to deploy the sensors in the field. We also thank all our previous team members Mr. Vinay, and Mr. Aswath kumar for their contributions to this project. We thank our department colleague Mr. Aswathnarayan Singh who has played a big role and support towards the deployment.

\section{REFERENCES}

[1] J Panchard, et al., ICTD2006, "COMMON-Sensenet:Improved Water Management for Resource-Poor Farmers via Sensor Networks" May 2006

[2] http://www.zyxel.com

[3] Crossbow technology, Inc "Mica2 Wireless Measurement system"

[4] T.V.Prabhakar and M.Aswathkumar, CEDT communication networks internal report-10 “ Mica2 Range measurement ". Jun 2005.

[5] T.V.Prabhakar and M.S.Sujay, CEDT communication networks internal report - 11 "An intelligent code for auto detection of sensors" Apr 2006.

[6] T.V.Prabhakar and M.S.Sujay, CEDT communication networks internal report-12 "Flash corruption in MICA2 motes in field deployment" Jul 2006.

[7] Decagon devices, Inc "The ECH20 Probe" Available at:

[8] http://www.ech2o.com/Ech20\%20probe.htm

[9] Shockfish SA,"Introducing Tinynode" [Online document] Available at: http://www.tinynode.com/

[10] P.R.Seshagiri Rao, H S Jamadagni, and Andre Pittet, CEDT

[11] Communication networks internal report -13 "Use of sensor data" Oct 2006.

[12] Sensirion, Inc. http://www.sensirion.com/ 